\title{
Socio-psychological characteristics of students as subjects of communication, depending on their attitude to mediated interaction
}

\author{
Anna Korochentseva, ${ }^{*}$ and Nelli Khachaturyan \\ Don State Technical University, 344000 Rostov-on-Don, Russia
}

\begin{abstract}
The article examines the problem of the relationship between the social and psychological characteristics of students as subjects of communication and their propensity for indirect communication, spending a significant part of their personal time in social networks. A comparative analysis of the severity of difficulties in a real act of communication among students who prefer direct (real) and mediated communication is carried out. The reliability of the results obtained is confirmed by analyzing the average values of the studied group indicators, and using the methods of mathematical statistics (correlation analysis, Student's t-test) and found a relationship between the propensity of students to communicate indirectly, the time spent communicating in social networks and the severity of difficulties in real interaction, caused by their specific socio-psychological characteristics as subjects of communication. The greatest difficulties in their views are associated with the influence on the emergence of difficulties in communication of such parameters as the intensity of communication, the number of partners, the presence of witnesses of communication, age, gender, status; suspicious, arrogant, disrespectful attitude during the conversation; social-perceptual and expressive-speech characteristics of partners; as well as the skills and abilities of the interlocutor regarding the organization of interaction. As the main conclusion, the researchers note that students who prefer real and mediated interaction have various psychological characteristics that characterize them as subjects of communication.
\end{abstract}

\section{Introduction}

In recent years, computer technologies and the global Internet have been actively developing. Now computers and smartphones are not a luxury item, but everyone has it. This widespread dissemination of technology has led to the emergence of a new form of communication - mediated communication, or online communication, which is communication using various technical devices: computer, smartphone, etc. $[1,2,3,4,5]$.

In modern studies, it is stated that the ratio of the number of real and mediated communication among modern students has changed greatly over the past decades (R. Verderber, K. Verderber, Romanov M.V., E.P. Belinskaya), at the same time, the authors

${ }^{*}$ Corresponding author: anna-kor@bk.ru 
emphasize the importance and necessity of real communication for the normal life of a person, the health of her psyche (G.M. Andreeva, R. Verderber, E.P. Belinskaya, O. A. Tikhomandritskaya, E.P. Belinskaya, Lukina N.A., Shakurova I.P.). The authors emphasize that communication covers all spheres of human life and society as a whole. It is the basis of life, since through it plans, feelings, desires, thoughts are realized, the attitude towards people around them and oneself in particular are shown. Andreeva G.M. notes that communication is an extremely delicate process of human interaction [6]. In communication, the individual characteristics of all participants in this process are revealed in the most diverse ways. Understanding of the essence of communication is revealed through such words as: contact, connection, interaction, exchange, method of unification.

For several centuries, people have communicated with each other directly, using all verbal and non-verbal means. In addition, letters, drawings, books and other objects were used to convey thoughts, ideas, emotions, feelings, experiences. Such communication is called - indirect or mediated. Recently, various computer technologies have closely invaded the communication process, which forced a serious revision of the structural and qualitative features of this process. In this regard, the term "mediated communication" has become popular.

Russian researchers note the negative impact on young people of uncontrolled being on the Internet, a change under the influence of this form of interaction of cognitive structures, while foreign ones more adhere to a utilitarian view of the online communication environment $[2,7,89,10,11,12,13,14,15,16]$.

The availability of the use of modern technical means, allows you to take a fresh look at the possibilities of meeting the basic needs characteristic of student age. It is in adolescence that a person especially needs communication, acceptance, support from another, and so that there is no criticism and condemnation. Through mediated communication, all of these needs can be satisfied. Thanks to the possibility of anonymous communication, accessibility and a sense of security, a person can form a trusting relationship, and in case of failure, you can always end the conversation. With indirect communication, difficulties arise with determining gender, age and social status, this is what allows you to create a new personality and realize your needs on its basis. Thanks to its ease and accessibility, mediated communication has become so popular $[1,2,3,14]$.

Modern researchers believe that mediated communication has little in common with real, and is quite specific [1, 2, 3, 4, 12]. A.G. Maklakov writes that mediated communication is communication in which there are other people, mechanisms, things (for example, a telephone conversation, a video conversation or a letter) [17]. Initially, mediated communication was associated with the use of special means and tools (pen, paper), writing, then television and radio, at the present stage of development of the society, we are mainly talking about a smartphone, a computer. That is, a smartphone and a computer have become frequent intermediaries in organizing communication and exchange of information.

One of the common forms of mediated communication is text message (chat). Almost everything that is the subject of direct communication with another person can be stated on paper. Thanks to modern technologies, namely the widespread use of the Internet, mediated communication has entered the life of a modern person, transforming into several new forms. These forms include: fast text messages, voice messages, video chat, as well as the ability to have a conversation with several or even hundreds of people at the same time.

Communication with the help of an intermediary (computer or telephone) has its own characteristics, but it is similar to the real one in that the interaction in it also takes place in the "person - person" system, and the computer is a link between these people, similar to the voice, body position, facial expressions in direct communication.

One of the clear features of mediated communication on the Internet is anonymity. The user can transform himself, take a different name, indicate a different gender, or change his 
appearance. There is also an opportunity not to indicate data about yourself at all, it is this opportunity that attracts users more and more.

Another important feature is the ability to leave any impression about yourself. In virtual reality, you can become whoever you want. You can embellish yourself, or you can create an alter ego. This fact also attracts the attention of scientists regarding what needs are satisfied by the subject hiding his "true self", and what psychological reasons led him to this $[4,5,6,7,8]$.

Voluntary of contacts and no fear of failure. With indirect communication, it is possible to start a dialogue with any person without experiencing discomfort, and in case of failure, you can stop the dialogue. In the modern world, people are often afraid to initiate communication with unfamiliar people, fearing to be not interesting and unattractive to another person. With direct contact, all this will be obvious due to various non-verbal manifestations (facial expressions, gaze, posture). Mediated communication makes it possible to exclude this part of the information flow.

Close to this parameter is the lack of the ability to see the interlocutor and be visible to him. In the case of an audio conversation (a phone call), it is possible to hear the interlocutor, but it is not possible to see him, thus only intonation remains from the entire spectrum of non-verbal communication. But even the presence of only this substructure of non-verbal communication is of great importance. Studies show that due to the intonation of callers who hear each other, dialogue is considered more constructive. When communicating in the form of SMS messages, information is absorbed worse, because the said phrase with different intonation can have different meanings [1, 2, 14].

This is the reason for the appearance of "emoticons" and other visual symbols in the structure of communication via a smartphone. Normally, if a person has nothing to hide, he has a strong need to express emotions and receive them from other people. The absence of such visual signs may indicate that the subject of communication does not want us to know about his emotional experiences.

A sense of security and impunity is another characteristic feature of online communication. Thanks to the ability to remain anonymous, there is a feeling of impunity and security, because it is difficult to calculate the user's address. This makes it possible to let out all the hidden desires and needs, to "take revenge" for all existing personal problems, as a rule, all these are unconscious processes. Such types of communication as "trolling" and "bullying" appear $[8,11]$. Another important point is the absence of strict social and culturally determined requirements for the subjects of communication. Thanks to the widespread use of the Internet, people with different views, people of different cultures and nationalities can participate in the dialogue. Such intercultural and social tolerance helps many to be themselves in their views on life and various events, without fear of negative prejudices.

Communication is an activity that is of particular value in adolescence. This is a way of spending leisure time, as well as a means of self-expression and establishing contacts with peers $[3,4,8,10]$. A person who knows how to competently build speech and communication shows a high level of development of his personality. The effectiveness of life and activity largely depends on the realization of individual and group capabilities of a person in communication. Communication is a special process that allows you to form a personality $[5,6,17]$.

Adolescence is a critical period in the process of personal evolution. From the age of 14, interpersonal relationships are being formed, in which attitudes toward the subjects of reality play a different role: toward the elderly, toward relatives, toward peers, toward teachers, toward friends, toward oneself, toward representatives of other races, religions and nationalities $[3,5,8,18,19,20]$. 
The psychological world of the adolescent period is turned to inner life, a young man is often closed, thoughtful, fantasizes a lot. The same period is marked by intolerance, irritability, and a tendency to aggression. The stage of self-knowledge and self-affirmation begins, young people become very critical in relation to reality, and a tendency to expand the unacceptable is gradually observed [15].

All of the above makes it possible to say that the psychological characteristics of young people as subjects of communication are being rebuilt and changed following the changes taking place in society. The modern generation differs significantly from the previous one, primarily in that it was brought up in a different "technical environment", and therefore has significant social and psychological characteristics specific to this generation. In this regard, the study of ideas about oneself as a subject of communication among students with different attitudes towards indirect communication, in our opinion, is of great interest.

\section{Materials and methods}

The purpose of the research was to study the idea of oneself as a subject of interaction among students with different attitudes towards indirect communication. The hypothesis of the study was the assumption that the idea of oneself as a subject of interaction will differ among students with different attitudes to real and mediated communication.

The methodological toolkit included the following questionnaires. To determine the propensity of students to mediate communication, we used a questionnaire developed by us on the basis of a theoretical analysis of scientific literature "Propensity to mediated communication" and the Kimberly Young test "For Internet addiction". 2. To determine the idea of oneself as a subject of communication and interaction, as well as sociopsychological characteristics that hinder real communication of students, the questionnaire "Socio-psychological characteristics of the subject of communication" (V.A. Labunskaya) was used. 3. The questionnaire, developed by us on the basis of the analysis of scientific literature, "The propensity of students to mediate communication." The empirical object of the study was 50 university students. 20 are female students, aged 17 to 24 , and 30 are male students, aged 17 to 25 . The reliability of the data obtained was ensured using such mathematical procedures as: calculating the significance of differences according to the Student's t-test, and determining the Pearson's rank correlation coefficient, using the computer program "SPSS 17.0 for Windows".

\section{Results}

First, we analyzed the propensity for indirect communication among students using a questionnaire developed by us, which allowed us to divide them into 2 groups.

The first group included students, whose propensity for real communication averaged 15.9, which indicates that the subjects choose real communicationto to a greater exten. Their propensity to communicate indirectly is 4.4. This indicates that, despite the preferences of this group for real communication, students note that mediated communication has its merits (for example, with mediated communication, the dialogue is easier to complete). We then tested these students for Internet addiction using the Kimberly-Young test. The obtained results showed that there is no dependence among students of this group. This is evidenced by the group average of 40, 9 points. That is, the subjects do not neglect household chores in order to spend more time on the Internet, their success does not suffer because of the time spent on the Internet, the subjects do not feel that without the Internet, life is empty and boring. 
In the group of students who prefer indirect communication, the average severity indicator on the scale of real communication is 7.9. This indicates that students of this group do not exclude real communication from their lives, but to a greater extent prefer mediated communication. They also showed signs of Internet addiction, as evidenced by the group average of 57.3. Subjects often make new acquaintances on the Internet, neglect household chores due to their addiction to the Internet, these students sacrifice sleep by staying up late in the network, they experience depression or nervousness when they are offline and note that this state goes away as soon as they find themselves on-line.

To determine the significance of the differences between the propensity of students to mediate or real communication, as well as Internet addiction, we used the Student's t-test. As a result, significant differences were found in the propensity for indirect or real communication $(t=-3.398)$. Students of group 2 have a significantly higher propensity for indirect communication than students of group 1.

Further, we investigated the social and psychological characteristics of students in two groups (group 1 - students who prefer real communication, group 2 - students who prefer indirect communication) as subjects of communication. For this we used the questionnaire "Socio-psychological characteristics of the subject of communication" V.A. Labunskaya. Based on the figure, it can be seen that the indicators of students of group 1, on the scales "Expressive - speech characteristics of partners" $(\mathrm{m}=8.52)$, "Social - perceptual characteristics of partners" $(\mathrm{m}=8.99)$, "Attitude - referrals of partners" $(\mathrm{m}=9.61)$, "Judgments revealing the skills and abilities of organizing interaction" $(\mathrm{m}=8.96)$, "The idea of the influence on the occurrence of communication difficulties of such parameters as the intensity of communication, the number of partners, the presence of witnesses of communication, age, gender, status " $(\mathrm{m}=8.29)$, have an average level, which indicates that these students do not experience difficulties associated with these aspects of communication.

The indicators of students of the 2 nd group on the scales have the following meanings: "Expressive - speech characteristics of partners" $(m=14.9)$ - a high level of severity, this suggests that, for example, a frozen posture, motionless facial expression or loud speech complicate the interaction between interlocutors. "Socio-perceptual characteristics of partners" $(m=15.12)$ - a high level of severity, this suggests that such qualities as the inability to correlate the actions of people with their personality traits, the desire to attribute people to a certain type, the habit of judging a person by his appearance makes it difficult to interact between interlocutors. "Attitude - referrals of partners" $(m=15.92)$ - a high level of severity, this scale suggests that difficulties in the communication process arise depending on how a person perceives another person's attitude towards him. "Judgments that reveal the skills and abilities of organizing interaction" $(m=13.96)$ - a high level of severity, this suggests that judgments such as the desire to speak more than to listen, the desire to impose one's point of view or the inability to get out of the conversation in time make the interaction between interlocutors difficult. "The idea of the influence on the occurrence of communication difficulties of such parameters as the intensity of communication, the number of partners, the presence of witnesses of communication, age, sex, status" ( $\mathrm{m}=$ 16.69) - a high level of severity. This scale includes: the state of health, mood, willingness to communicate with the conversation partner influence and complicate interaction in the communication process. 
Table 1. Indicators of the severity of difficulties among students as subjects of communication, preferring real or indirect interaction with a partner

\begin{tabular}{|c|c|c|c|c|c|}
\hline \multirow{2}{*}{ Scale } & \multicolumn{2}{|c|}{ Group 1 } & \multicolumn{2}{c|}{ Group 2 } & \multirow{2}{*}{ Max } \\
\cline { 2 - 5 } & Indicator & $\begin{array}{c}\text { Severity } \\
\text { level }\end{array}$ & Indicator & $\begin{array}{c}\text { Severity } \\
\text { level }\end{array}$ & \\
\hline ES expressive-speech & 8.52 & medium & 14.9 & high & 20 \\
\hline SP social-perceptive & 8.99 & medium & 15.12 & high & 20 \\
\hline RT Relationship-treatment & 9.61 & medium & 15.92 & high & 20 \\
\hline SA skills and abilities & 8.96 & medium & 13.96 & high & 20 \\
\hline IO influence of occurence & 8.29 & medium & 16.69 & high & 20 \\
\hline
\end{tabular}

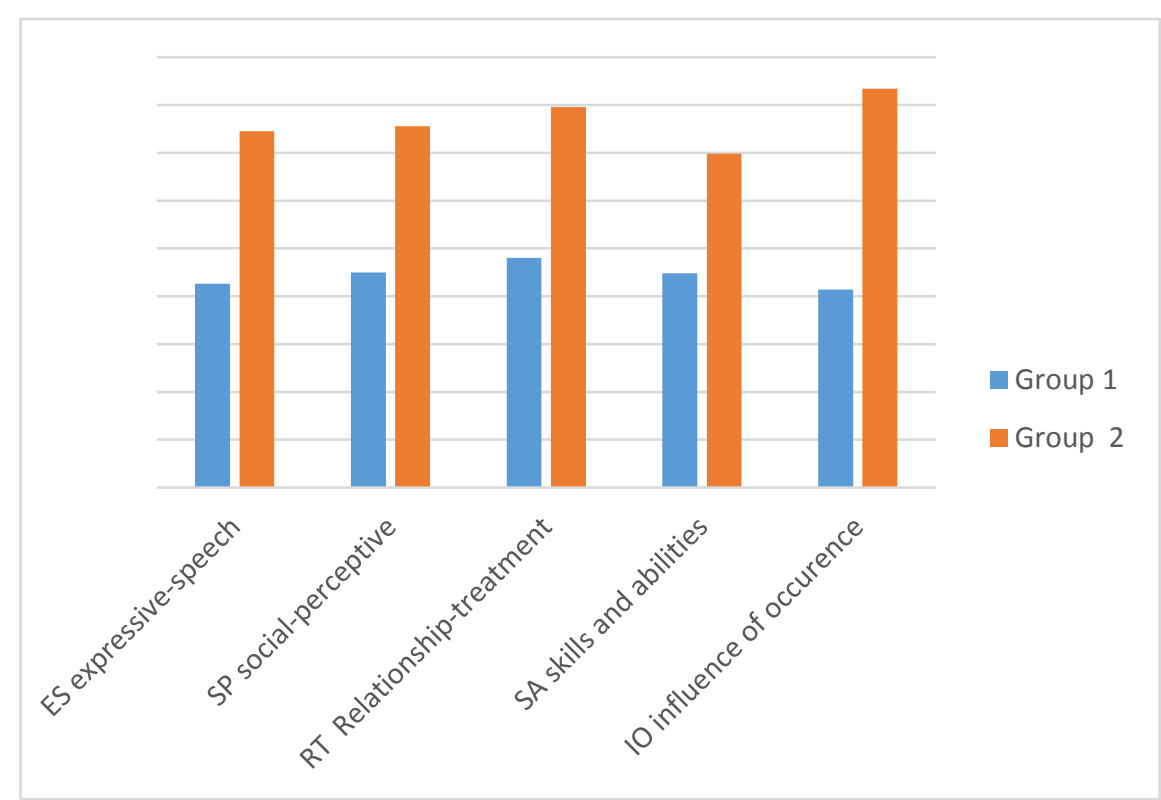

Fig. 1. Indicators of the severity of difficulties among students as subjects of communication, preferring real or indirect interaction with a partner

To identify the significance of differences in the socio - psychological characteristics of students as subjects of communication, such mathematical procedure as the Pearson's rank correlation coefficient was used. As a result, significant relationships were found between the frequency of the student's choice of mediated communication and the idea of himself as a subject of communication, which consists in the fact that the more a student is inclined to make a choice in favor of mediated communication, the more he will experience difficulties in real communication in the following scales: "Expressive speech characteristics of the partner" ( $\left.\mathrm{r}=, 711^{* *}, \mathrm{p} \geq 0.05\right)$; "Socio-perceptual characteristics" $(\mathrm{r}=, 703 * *, \mathrm{p} \geq 0.05)$; "Treatment relations" ( $\mathrm{r}=, 709 * *, \mathrm{p} \geq 0.05)$; "Skills of organizing interaction" $(\mathrm{r}=, 622 * *$, $\mathrm{p} \geq 0.05)$; "The influence of the occurrence of difficulties" ( $r=, 773 * *, p \geq 0.05)$. This means that factors such as the intensity of communication, the number of partners in the dialogue, age, gender and status of the interlocutor, significantly complicate the interaction with the communication partner for these students.

\section{Discussion}

The study found significant relationships between the propensity of students to communicate indirectly and self-image as a subject of communication. The higher the 
indicator of the propensity for indirect communication, the more difficulties a given student experiences as a subject of communication in a real situation of interaction. These difficulties relate to sensitivity to certain aspects of communication. The greatest difficulties are observed on a scale that includes the idea of the influence on the occurrence of difficulties in communication of such parameters as the intensity of communication, the number of partners, the presence of witnesses of communication, age, gender, status. It is interesting that it is these characteristics that lose their significance in the process of mediated communication. In addition, the identified difficulties among students who prefer indirect communication related to the scales: including judgments describing the "relationship-treatment" of partners to each other (for example, suspicious, arrogant, disrespectful attitude in the process of a conversation, or excessive desire of the interlocutor to make the right impression); social and perceptual characteristics of partners (include the social side of communication and understanding by partners of each other, for example, not the ability to put oneself in the place of another, the inability to "read" the feelings and intentions of another person or "mistakes" of the partner in assessing the feelings and moods of another person).

To a less extent, but also strongly expressed difficulties related to the expressive-speech characteristics of the partner (include the emotional coloring of the behavior and speech of the communication partner, demonstrating the relationship between the communicators), as well as a group of judgments that reveal the skills and abilities of organizing interaction (ability or inability to competently conduct a conversation, tactfully and without interrupting the interlocutor). Interestingly, most of the identified characteristics that cause difficulties in communication lose their significance in the process of mediated communication.

A characteristic feature of students who choose real communication is that they are not afraid to be funny in the eyes of others, such students can communicate with people of different gender and status, they are able to put themselves in the place of another person and are better oriented in various communication situations and interactions.

\section{Conclusion}

The emergence of a new form of communication entailed changes in human life, namely, a new form of interaction appeared - mediated communication. Such communication has a number of significant differences from direct (real) communication. For example, a large audience, the removal of rigid social boundaries, the ability to correct messages (which is impossible in direct communication, since words cannot be taken back), the invisibility of the interlocutor, anonymity and, as a result, a sense of security, the ability to leave any desired impression about yourself.

In addition, the authors note the unregulated behavior, a decrease in the forms of interaction, there is an increase in social and psychological risk of communication, as well as compensatory virtual emotionality. Psychological characteristics of a person as a subject of communication also undergo changes. In our study, it was shown that in subjects prone to indirect communication and spending a significant amount of their time in social networks, one can state the presence of difficulties in a situation of real, direct communication. Significant relationships were established between the propensity of students to communicate indirectly and the severity of various difficulties in a real act of communication.

Thus, all of the above allows us to say that the long-term involvement of students in indirect forms of interaction has a significant impact on their idea of themselves as a subject of real communication, and leads to difficulties in real, direct communication. 


\section{References}

1. D. Bylieva, V.Lobatyuk, A. Safonova, Humanities and Social Sciences Reviews 7 (1), 332-340 (2019)ю doi: 10.18510/hssr.2019.7138

2. I.S. Shapovalova, Youth communication strategies: communication without borders and site traps. Culture and Education: Social Transformations and Multicultural Communication, Proceedings of the Middle-Term Conference RC04 Sociology of Education International Sociological Association (ISA), PUDN University, 110-117 (2019)

3. A.Y. Petukhov, S.A. Polevaya, N.S. Chuprakova, Influence of the modern web communication on the psychological characteristics of the rising generation (12-13 year old) from the view of the information images theory, Procedia Computer Science. "7th Annual International Conference on Biologically Inspired Cognitive Architectures, BICA 2016", 423-428 (2016). doi: 10.1016/j.procs.2016.07.459

4. V.V. Smirnov, Higher education today 4, 72-76 (2019). doi: 10.25586 / RNU.HET.19.04.P.72

5. O.A. Tikhomandritskaya, N.G. Malysheva, Z.D. Shaekhov, Contemporary research on psychological well-being in the digital generation (In the book: Digital Society in a Cultural-Historical Paradigm. Collective monograph. Edited by T.D. Martsinkovskaya, V.R. Orestova, O.V. Gavrichenko. Moscow, 2019) https://www.elibrary.ru/item.asp?id=37182518

6. G.M. Andreeva, Psychological research 5 (26), (2012). https://www.elibrary.ru/item.asp?id=19068156.

7. M.I. Wolf, A.S. Sukhanova, Features of interpersonal relationships in the Internet communication and anxiety of students, In the collection: Self-realization of personality in the modern world, Materials of the All-Russian scientific-practical conference with international participation, Scientific editor S.S. Kudinov, 44-49 (2018) https://www.elibrary.ru/item.asp?id=35360527.

8. A.A. Klyukina, Cyberbullying as a threat to the psychological health of the personality of adolescents and young men, Psychology of personality (Collection of scientific papers, Magnitogorsk, 2019) https://www.elibrary.ru/item.asp?id=38027327.

9. D.N. Karpova, D.V. Goloukhova, Communicology 4 (3), 159-168 (2016) https://www.elibrary.ru/item.asp?id=26323270.

10. D.N. Karpova, Russian Society of Sociologists. 6152-6159 (2016) https://www.elibrary.ru/item.asp?id=27501921.

11. V. Vereva, Russian Literature 118, 107-140 (2020). doi.org/10.1016/j.ruslit.2020.11.005

12. L.A. Buraeva, Z.I. Dadova, Socio-political sciences. 6, 90-93 (2019) https://www.elibrary.ru/item.asp?id=41747408.

13. Hongyee Chan Gloria, Computers in Human Behavior 111, 106407 (2020). doi.org/10.1016/j.chb.2020.106407.

14. R. Valaitis, J Med. Internet. Res 7 (5), 51 (2005). doi:10.2196/jmir.7.5.e51.

15. D. Dautov, A. Korochentseva, K.M. Mohamed, SHS Web of Conferences. 70, 06001 (2019). doi: 10.1051/shsconf/20197006001.

16. V.I. Pishchik, G.A. Molokhina, E.A. Petrenko, Y.V. Milova, International Journal of Cognitive Research in Science, Engineering and Education (IJCRSEE) 7 (2), 67-76 (2019). doi.org/10.5937/IJCRSEE1902067P. 
17. A.G. Maklakov, I.A. Sidorova, Topical issues of developmental psychology and personality formation: methodology, theory and practice, Collection of materials of the All-Russian scientific-practical conference. 130-134 (2018) https://www.elibrary.ru/item.asp?id=36719777.

18. G. Kozhukhar, A. Belousova, E. Breus, E3S Web Conf. Innovative Technologies in Science and Education 210, (2020). doi.org/10.1051/e3sconf/202021018009.

19. E. Suroedova, Y. Tushnova, E3S Web of Conferences 210, 19004 (2020). doi.org/10.1051/e3sconf/202021019004.

20. V. Pishchik, Z. Spivachuk, The uniqueness of values and personal traits of Generation $Z$ students in the innovative educational space of the southern region of Russia, Innovative Technologies in Science and Education (ITSE-2020), E3S Web Conf 210, 18035 (2020). doi.org/10.1051/e3sconf/202021018035. 